\title{
EC relevant programmes for research and exploitation of results
}

\author{
Viviane Thévenin \\ Commission of the European Communities DG XII, rue de la Loi 200, B-1049 Brussels, Belgium
}

Accepted 7 April 1994

One of the workshops organized during the ESEM Conference on Engineering and Medicine (Stuttgart, 25-28 April 1993) was dedicated to this theme. The following three papers correspond to three out of five oral presentations which were made at the workshop; the first two, which are not included here, dealt with Telematics applied to health care, better known as the AIM (Advanced Informatics in Medicine) programme of the EC, and with the EC Biomedical and Health Research programme. These two EC research programmes have been presented and discussed elsewhere, and accordingly are better known now among the interested scientific community in Europe.

In that workshop, the interesting aspect was to present some possibilities for support which are offered by the EC downstream, once research has been successful enough to lead to possibilities for industrial development and marketing, which is compulsory if any innovative medical device or drug is to be applied in the health care system. It had been thought interesting to introduce such possibilities and discuss them within the Stuttgart conference, because, as is often noted, whereas in Europe, research is quite productive, in terms of innovative ideas, the transfer to industrial development frequently raises difficulties, often reaching situations in which innovations originating in Europe are industrially developed elsewhere in the world and re-introduced in the European health care system. One of the elements which is often quoted to explain such situations is the difficulty of raising venture capital in Europe. Two of the following papers aim at providing some information on such a point:

- the first one, by Deborah Lambrechts, deals with venture capital companies and the EUROTECH capital programme of the EC;

- the second one, by Ailie Menzies, describes more specifically the EC EUROTECH INNVEST service.

The EUROTECH capital programme aims to seek out the projects of new companies and to increase the chances of success of entrepreneurs applying for venture capital funding. It describes the relationship between entrepreneurs and venture capitalists, the criteria governing venture capital investment and the importance of adequate presentation of projects to venture capitalists.

The EUROTECH INNVEST service aims at identifying small and medium sized enterprises requiring finance which are involved in high technology developments, select those suitable and present them to investors; this service in fact provides companies with a direct line to potential investment.

The last paper was aimed at providing a national perspective beside the EC one. The Dutch experience of supporting medical technology research describes efforts made by a national gov- 
ernment to stimulate such research by specific policies. Dr. van der Torren interestingly shows the major problems which were identified during the implementation of these policies in the Netherlands, but it is obvious that such problems are commonly found in other European countries, namely:

- the need for more information about the market to reduce existing obstacles to the sale of products and services, and to enable opportunities to be identified more easily;

- the inefficient application of expertise due to the lack of proper cooperation between research institutes, industry and the health care sector;
- the complex relationships and interests in the health care sector, which could impede the identification of new market opportunities and the innovative drive within industry.

It is hoped that these papers, by making scientists more aware of such possibilities and their relevant requirements will contribute to a better knowledge about the financial community, and thereby contribute to facilitate the transfer of research ideas to industrial applications, although it is obvious that this transfer process is quite complex and that other actors, apart from scientists and engineers are involved. 